\title{
Percepções do fonoaudiólogo recém-formado quanto \\ a sua formação, intenção profissional e atualização de conhecimentos
}

\author{
Perceptions of newly graduated Speech and Language \\ Pathologists regarding their academic formation, professional \\ goals and knowledge update
}

Denise Guapyassú Meirelles da Silva ${ }^{1}$, Tania Maria Marinho Sampaio² ${ }^{2}$ Esther Mandelbaum Gonçalves Bianchini ${ }^{3}$

RESUMO

\begin{abstract}
Objetivo: Investigar as percepções do fonoaudiólogo recém-formado do estado do Rio de Janeiro quanto a sua formação, intenção profissional e busca por atualização de conhecimento. Métodos: Participaram fonoaudiólogos graduados em cursos de formação no estado do Rio de Janeiro, nos anos 2005 e 2006. Foi aplicado um questionário constituído por dez perguntas fechadas, elaboradas para esta pesquisa e previamente verificadas por projeto piloto. Os dados foram submetidos a análise estatística com cruzamento de variáveis. Resultados: Observou-se que a maioria dos fonoaudiólogos considerou-se atendida em suas expectativas e segura dos conhecimentos adquiridos $(\mathrm{p}<0,001)$. Esses profissionais pretendem atuar principalmente em clínicas $(89 \%)$ e continuar se atualizando por meio de cursos de especialização (70\%). A área de Linguagem foi considerada a que melhor subsidiou sua formação (68\%), enquanto que Saúde Coletiva foi a que menos atendeu às expectativas (72\%), com diferenças estatisticamente significativas. A indústria (70\%) foi o local de trabalho considerado de acesso mais difícil ao fonoaudiólogo ( $<<0,05)$. Conclusão: Os fonoaudiólogos do estado do Rio de Janeiro graduados em 2005 e 2006 consideraram-se atendidos em suas expectativas quanto à formação, especialmente no que se refere à área de Linguagem, e apontaram maior interesse por atuação em clínica. Quanto à busca por atualização de conhecimento, a especialização foi o curso que despertou maior interesse.
\end{abstract}

Descritores: Capacitação profissional; Fonoaudiologia/educação; Educação continuada; Mercado de trabalho

\section{INTRODUÇÃO}

O processo de formação acadêmica vem assumindo maior importância nos últimos anos, principalmente em função das várias alterações que as propostas curriculares buscam trazer aos cursos de graduação ${ }^{(1)}$. Especialmente relevantes são as discussões que apontam para as transformações pelas quais vêm passando as sociedades atuais, nas quais

Trabalho realizado no Curso de Mestrado Profissionalizante em Fonoaudiologia da Universidade Veiga de Almeida - UVA - Rio de Janeiro (RJ), Brasil.

(1) Mestre, Fonoaudióloga clínica - Rio de Janeiro (RJ), Brasil.

(2) Doutora, Professora Adjunto do Curso de Mestrado Profissionalizante em Fonoaudiologia da Universidade Veiga de Almeida - UVA - Rio de Janeiro (RJ), Brasil.

(3) Doutora, Professora Adjunto do Curso de Mestrado Profissionalizante em Fonoaudiologia da Universidade Veiga de Almeida - UVA - Rio de Janeiro (RJ), Brasil.

Endereço para correspondência: Denise Guapyassú. Av. Afonso Arinos de Melo Franco, 285/2001, Barra da Tijuca, Rio de Janeiro (RJ), Brasil, CEP:

22631-455. E-mail: deniseguapy@globo.com

Recebido em: 28/7/2008; Aceito em: 8/8/2009 fenômenos de globalização são fatos presentes que fazem surgir uma multiplicidade cada vez maior de referências a serem inseridas nos campos curriculares, tornando difícil a sua delimitação. Nesse contexto, faz-se necessário que sejam estabelecidas análises reflexivas sobre as estruturas curriculares, a fim de se privilegiar uma identificação entre o conhecimento científico e a atividade que o profissional desenvolverá no seu cotidiano.

A formação do fonoaudiólogo tem requerido um arsenal de competências cada vez mais complexo, que, por um lado, deve contemplar as adversidades típicas de uma população carente ainda de atendimento apropriado para as suas demandas e, por outro lado, a sua crescente atualização frente aos avanços tecnológicos no cuidado com a saúde. O papel dos cursos de graduação deve ser cada vez mais estratégico. Contudo, a concepção e o desenvolvimento de tais cursos podem não estar privilegiando, em sua totalidade, os aspectos relevantes para um bom desempenho profissional. Nesse sentido a busca por permanente atualização do conhecimento torna-se parte integrante na consolidação da bagagem acadêmica, com o 
engajamento do profissional na busca incessante por novos conhecimentos em sua área ${ }^{(2)}$.

No início dos anos 80, foi apresentada uma ampla pesquisa abrangendo todos os continentes: Europa, América Latina, América do Norte, África, Ásia e Oceania ${ }^{(3)}$, na qual foram apontadas três categorias básicas referentes à variação do perfil desse profissional: a primeira, composta por profissionais que são formados para atuar na educação especial; a segunda refere-se àqueles que trabalham no campo da medicina dentro de hospitais; finalmente, a terceira categoria, composta por profissionais formados para atuarem em consultórios, quanto à avaliação e tratamento da fala, voz e alterações de linguagem ${ }^{(3)}$. Essas categorias, apesar de terem sido apontadas no início da década de 80 , foram aceitas durante diversos anos e passaram a incluir outros olhares, a partir da atuação multidisciplinar, tanto no que se refere à avaliação, quanto ao tratamento, propiciando uma reflexão sobre a formação e a modificação desse profissional.

No Brasil, as práticas fonoaudiológicas estiveram inicialmente a serviço da padronização da língua nacional ${ }^{(4)}$, sendo que o $1^{\circ}$ Congresso da Língua Nacional (First Congress of the National Language) foi considerado por escritores, poetas, músicos, artistas e cientistas como o marco inicial da Fonoaudiologia no nosso país ${ }^{(5)}$. Na década de 90 o número de autores e de publicações brasileiras aumentou substancialmente na literatura científica internacional e, apesar de os fundos governamentais terem sido reduzidos em mais de $70 \%$, a comunidade científica brasileira expandiu sua produção ${ }^{(6)}$.

A formação educacional do fonoaudiólogo brasileiro foi amplamente descrita em publicação no ano de $2006^{(7)}$. Esse estudo também apontou para o fato de o Brasil ser responsável por $1 \%$ de toda publicação científica no mundo e evidencia a necessidade de melhor distribuição de profissionais em território brasileiro ${ }^{(7)}$ uma vez que, de acordo com os dados do Conselho Federal de Fonoaudiologia (CFFa) citados nesse trabalho, há 25.000 fonoaudiólogos registrados nos sete Conselhos Regionais, sendo que 58\% desses profissionais atuam no Rio de Janeiro e em São Paulo.

Estudos regionalizados anteriores a 2006 podem ser encontrados na literatura. No final da década de 90 , um importante estudo traçou o perfil do fonoaudiólogo no estado de São Paulo, e suas conclusões mostraram a relevância de se realizarem pesquisas similares nos demais estados do Brasil ${ }^{(8)}$. Somente quatro anos depois, tal área de conhecimento parece ter despertado um maior interesse dos pesquisadores. A partir de 2001, a literatura foi contemplada com um número relativamente significativo de estudos que se propuseram a pesquisar o perfil do fonoaudiólogo enfocando cidades, estados ou regiões do sul, sudeste e nordeste brasileiros ${ }^{(9-11)}$. Embora com o mesmo objetivo, as metodologias e conclusões dos trabalhos guardam suas particularidades.

No estudo referente a São Paulo, realizado em $1997^{(8)}$, consta que entre os $88,86 \%$ dos profissionais ali atuantes, $37 \%$ apresentavam cursos de especialização (pós-graduação lato sensu) e $(6,66 \%)$ apresentavam mestrado e/ou doutorado (pós-graduação stricto sensu). Em termos de atividade profissional, a pesquisa mostrou que $12,26 \%$ atuavam na área de Audiologia e 63,07\% na área de Linguagem. Vale ressaltar que, na metodologia aplicada a esse trabalho, os autores incluíram as áreas de Voz e Motricidade Orofacial na área de Linguagem. Foi também observado que o fonoaudiólogo do interior do estado atuava, em maior concentração, no setor privado filantrópico e no setor público, enquanto que na região metropolitana, o maior número se concentrava no setor privado lucrativo (consultórios, clínicas particulares e universidades particulares). O trabalho concluiu que o fonoaudiólogo típico do estado de São Paulo era autônomo e trabalhava em consultórios e clínicas particulares.

O estudo da região sul do Brasil $^{(9)}$, realizado em 2001, investigou 1196 profissionais atuantes nos estados do Paraná (PR), Santa Catarina (SC) e Rio Grande do Sul (RS). Os autores mostraram que 77,5\% atuavam em mais de uma área, e $22,5 \%$, apenas em uma. Consultórios e clínicas particulares abrangiam 59\% dos profissionais da área.

O estudo realizado em São José dos Campos (SP) ${ }^{(10)}$, em 2004, mostrou que 78\% dos profissionais eram autônomos e trabalhavam em consultórios ou clínicas particulares. Apesar de 93\% terem feito especialização, principalmente em Motricidade Orofacial e Audiologia, o fonoaudiólogo dessa cidade continua atendendo a mais de uma área, devido ao tipo de demanda.

A região nordeste do país foi contemplada em uma pesquisa realizada no estado da Paraíba ${ }^{(11)}$, em 2005 . O perfil encontrado mostrou que $28,4 \%$ cursaram uma única especialização e, apesar disso, $58,2 \%$ atuavam simultaneamente em até quatro especialidades. Nenhum dos participantes da pesquisa possuía formação de pós-graduação (stricto sensu).

A literatura mostra algumas pesquisas com o objetivo de traçar o perfil do fonoaudiólogo, no entanto, como tais estudos são caracteristicamente regionalizados, diversas regiões do Brasil permanecem sem qualquer tipo de pesquisa. Embora o estado do Rio de Janeiro represente historicamente um dos centros de referência nas formações em diversas ciências, até a presente data não foi encontrado qualquer trabalho que tenha abordado o início da profissionalização e as percepções do fonoaudiólogo do Rio de Janeiro, focalizando a transição entre a formação universitária e o ingresso no mercado de trabalho. Da mesma forma, não foram encontrados trabalhos nesse estado que mostrassem se a formação adquirida no decorrer do curso de Fonoaudiologia teria privilegiado todas as áreas que necessariamente subsidiam a atuação profissional, assim como a intenção de aprofundamento por parte desses profissionais.

Nesse sentido, o presente estudo teve por objetivo investigar as percepções do fonoaudiólogo recém-formado do estado do Rio de Janeiro quanto a sua formação, intenção profissional e busca por atualização de conhecimento.

\section{MÉTODOS}

Esta pesquisa foi realizada após processos éticos pertinentes, constando da análise e aprovação dos procedimentos pela Comissão de Ética em Pesquisa da Universidade Veiga de Almeida (processo $n^{\circ} 75 / 07$ ).

Responderam ao questionário 263 fonoaudiólogos graduados nos anos de 2005 e 2006, oriundos de todos os 
cursos de graduação em Fonoaudiologia do estado do Rio de Janeiro, a saber: Centro Universitário Moacyr Sreder Bastos, Escola Superior de Ensino Helena Antipoff, Faculdade Redentor, Uni IBMR, Universidade Iguaçu, Universidade Federal do Rio de Janeiro, Universidade Estácio de Sá, Universidade Veiga de Almeida e Universidade Católica de Petrópolis, sendo que esta não oferece atualmente o curso de Fonoaudiologia.

Buscando-se obter o maior número possível de participantes, e contando com a colaboração do Conselho Regional de Fonoaudiologia $-1^{\mathrm{a}}$ região (CRFa1), foram realizados diversos procedimentos visando ao contato com os profissionais. O Conselho Regional de Fonoaudiologia - $1^{\text {a }}$ região realizou três chamadas, via internet ${ }^{(12)}$, para recrutar a participação, nessa pesquisa, dos fonoaudiólogos graduados em 2005 e 2006, inscritos no Conselho. Devido ao número inicialmente reduzido de adesão, foi também realizado contato com todas as universidades, solicitando dados em relação ao número de formandos no período pesquisado, além de contato direto com professores das instituições, com o objetivo de localizar os formandos (endereço, telefone ou e-mail). De abril a setembro de 2007, foi realizado contato com os alunos que responderam ao convite demonstrando interesse em participar da pesquisa.

Concordaram em participar 136 (52\%) fonoaudiólogos, que enviaram o questionário respondido até 30 de setembro de 2007, constituindo-se, assim, o grupo de pesquisa. Visando à caracterização do grupo, foram solicitadas informações quanto à faixa etária e formação de graduação referente à instituição em que realizou o curso e ao ano de conclusão.

O instrumento de pesquisa foi um questionário composto de 10 perguntas objetivas, com respostas fechadas, desenvolvidas pelas autoras para este trabalho. Tais questões abrangeram itens quanto à satisfação e expectativas em relação ao curso; segurança em relação aos conhecimentos adquiridos; segmentos em que pretende atuar; áreas que melhor subsidiaram a formação; áreas que não atenderam às expectativas; relação entre aulas teóricas e práticas; pretensão em continuar se atualizando e em que nível; verificação quanto a estar atuando profissionalmente e que ambientes de trabalho apresentam-se como de mais difícil acesso.

Com o objetivo de testar a adequação do questionário, foi aplicado previamente um projeto piloto, no qual 25 fonoaudiólogos atuantes responderam às dez questões, sem que fossem dados quaisquer esclarecimentos prévios. Após o preenchimento e devolução dos questionários, foi realizada entrevista individual com os participantes do instrumento piloto, visando a obter informações a respeito das eventuais dúvidas encontradas. A partir de então, as questões foram reformuladas, ajustadas e retestadas, resultando no questionário que compõe o material aplicado nesta pesquisa. Com base nas informações oriundas do procedimento piloto do material disponível de trabalhos anteriores e da Classificação Brasileira de Procedimentos em Fonoaudiologia ${ }^{(13)}$, foram definidos os itens de algumas questões quanto a:

- segmentos de atuação, designados como prevenção (incluindo-se promoção de saúde), pesquisa, empresarial, perícia, clínica (referente a processos de avaliação, diagnóstico e reabilitação fonoaudiológica), docência, assistência fonoaudiológica hospitalar e escolar (orientações fonoaudiológicas a instituições educacionais);

- áreas de atuação fonoaudiológica, designadas com base nas especialidades reconhecidas pelo $\mathrm{CFFa}^{(14)}$ : Voz, Audição, Linguagem, Motricidade Orofacial e Saúde Coletiva;

- locais de atuação, designados quanto aos locais de trabalho como hospitais, empresas, indústrias, escolas, unidades básicas de saúde, consultórios ou clínicas interdisciplinares e universidades ou faculdades (instituições de ensino superior).

Para assegurar a correta interpretação das questões, os participantes tiveram acesso a esclarecimentos para o preenchimento do questionário, constando de orientações quanto aos itens acima descritos, sempre que necessário.

Os participantes receberam o questionário por e-mail, foram instruídos a preenche-lo e reenviá-lo para as autoras. Os dados obtidos a partir dos questionários foram tabulados e submetidos a estudo estatístico analítico.

A análise estatística constou dos seguintes aspectos:

- a verificação da homogeneidade da distribuição proporcional das categorias das variáveis foi realizada por meio do teste de homogeneidade do qui-quadrado, quando se referiam às politomias ou pelo teste binomial, quando dicotômicas. No caso das politomias, a análise da diferença entre proporções de categorias foi realizada pelo teste de hipótese de diferença entre proporções dependentes: Q-Cochran e McNemar.

- a análise da associação entre variáveis foi realizada por meio do teste de independência das variáveis consideradas, pela utilização da estatística do Qui-quadrado, e pelo teste exato de Fisher.

Foi utilizado o intervalo de confiança de $95 \%$, sendo que as decisões estatísticas foram referidas ao nível de significância de $\mathrm{p}=0,05(5 \%)$.

\section{RESULTADOS}

Os resultados mostraram que grande parte dos fonoaudiólogos pretende atuar na clínica. Os segmentos prevenção, docência e assistência escolar formam o segundo conjunto de maior pretensão dos fonoaudiólogos, uma vez que as proporções entre os três segmentos mostraram-se sem diferença estatística significativa $(p>0,05)$. Os segmentos assistência hospitalar, pesquisa e empresarial formam o terceiro grupo na pretensão dos fonoaudiólogos, embora haja diferença estatisticamente significativa $(\mathrm{p}<0,05)$ maior para o segmento hospitalar do que para o segmento empresarial e pesquisa, os quais aparecem como estatisticamente equivalentes $(\mathrm{p}>0,05)$. Destoando de todos os demais segmentos, a perícia se apresenta como o menos pretendido $(\mathrm{p}<0,0001)$.

Quanto às áreas que melhor subsidiaram a formação, os dados mostram a Linguagem como a mais apontada, com diferenças estatisticamente significativas $(\mathrm{p}<0,0001)$. As áreas de Motricidade Orofacial e Audição formam o segundo conjunto apontado como de maior subsídio na formação profissional dos fonoaudiólogos, uma vez que as proporções entre essas duas áreas mostraram-se sem diferença estatística significativa entre si $(p>0,05)$, mas diferiram significativamente 
Tabela 1. Cruzamento de dados entre as variáveis expectativa e segurança

\begin{tabular}{lcccc}
\hline Expectativa de ser atendido & \multicolumn{4}{c}{ Segurança para atuar } \\
\cline { 2 - 5 } & Seguro/sozinho & Seguro/supervisionado & Inseguro & Total \\
\hline Parcialmente & 36 & 30 & 10 & 76 \\
Totalmente & 33 & 27 & 0 & 60 \\
Não atendeu & 0 & 0 & 0 & 0 \\
\hline Total & 69 & 57 & 10 & 136 \\
\hline
\end{tabular}

Teste de associação do Qui-quadrado: $\chi^{2}=8,524 ;$ g.I. $=2 ; p=0,014$

$(\mathrm{p}<0,05)$ das demais áreas consideradas no questionário. A área de Voz constituiu o terceiro grupo mais apontado quanto a esse item. Diferindo das demais áreas, a Saúde Coletiva foi apontada como a que menos subsídio trouxe para a formação do fonoaudiólogo $(\mathrm{p}<0,0001)$.

Continuar se atualizando é uma decisão quase unânime no grupo pesquisado $(\mathrm{p}<0,0001)$, podendo ser observada preferência diferenciada para cada nível de atualização do conhecimento $(\mathrm{Q}=146,2 ; \mathrm{g} .1 .=3 ; \mathrm{p}<0,0001)$. A maior parte dos fonoaudiólogos indicou a especialização como o mais pretendido (70\%), seguido do mestrado (61\%), da atualização (30\%) e do doutorado (26\%).

A maioria dos integrantes da amostra já se encontrava em atividade profissional na ocasião da pesquisa $(\mathrm{p}<0,0001)$.

Quanto aos locais de trabalho, os fonoaudiólogos apontaram as indústrias e as empresas como os de acesso mais difícil. Com diferença estatisticamente significativa $(\mathrm{p}<0,05)$ em relação às indústrias e às empresas, os fonoaudiólogos posicionam em segundo lugar os hospitais e as unidades básicas de saúde. Em terceiro lugar, o conjunto formado pelas universidades e escolas, com equivalência estatisticamente significativa $(\mathrm{p}>0,05)$. Seguem-se as clínicas e, por último, os consultórios.

Foi investigada a hipótese da existência de relação entre as expectativas do sujeito quanto ao curso realizado e a segurança em atuar frente aos conhecimentos adquiridos visando testar a hipótese de que o profissional satisfeito com curso realizado apresenta maior segurança na atuação profissional. Os resultados mostraram a associação entre as duas variáveis $(\mathrm{p}<0,05)$ confirmando a hipótese levantada (Tabela 1).

A hipótese de que fonoaudiólogos atuantes profissionalmente percebem a necessidade de estar continuamente em formação complementar de atualização foi igualmente testada. Os resultados mostraram uma associação positiva, porém de fraca intensidade (coeficiente $\mathrm{Phi}=0,397 ; \mathrm{p}=0,0005$ ), apontando para o fato de que àqueles profissionais que se encontram no mercado de trabalho pretendem aprofondar e atualizar seus conhecimentos profssionais (Tabela 2).
Tabela 2. Cruzamento de dados entre a variável continuidade de atualização e atuação profissional

\begin{tabular}{lccc}
\hline Continuar se & \multicolumn{3}{c}{ Atuando profissionalmente } \\
\cline { 2 - 4 } atualizando & Sim & Não & Total \\
\hline Sim & 119 & 14 & 133 \\
Não & 0 & 3 & 3 \\
\hline Total & 119 & 17 & 136 \\
\hline
\end{tabular}

Teste de McNemar: $p<0,01$ (coeficiente $P h i=0,397 ; p=0,0005$ )

A associação entre a satisfação com as aulas teóricas e práticas e a segurança para atuar (Tabela 3) mostrou-se estatisticamente significativa $\left(\chi^{2}=5,991 ; \mathrm{g} .1 .=2 ; \mathrm{p}=0,050\right)$, ou seja, os sujeitos que atribuem relação satisfatória entre as aulas têm mais segurança para atuar.

\section{DISCUSSÃO}

Os trabalhos correlatos apresentados na literatura apontam basicamente o levantamento das instituições, ano de conclusão e caracterização de sua atuação, analisando a continuidade e perfil do profissional já atuante ${ }^{(8-11)}$. O presente trabalho buscou investigar as percepções do fonoaudiólogo recémformado, do estado do Rio de Janeiro, quanto à sua formação, intenção profissional e busca por atualização do conhecimento. Para a compreensão global dos resultados deste estudo, é importante observar que se buscou apresentar a visão do fonoaudiólogo quanto às características dos cursos realizados, independente da instituição formadora, e sua pretensão de atuação, independente das oportunidades oferecidas pelo mercado de trabalho. Trata-se de uma pesquisa de opinião do fonoaudiólogo recém-formado e seus desejos de atuação, sem a preocupação de suas opções estarem influenciadas pela viabilidade de emprego na área desejada.

Os dados referentes à impressão dos fonoaudiólogos com relação ao curso realizado apontam que esses profissionais consideram suas expectativas atendidas, sentindo-se seguros em relação aos conhecimentos adquiridos. A relação entre

Tabela 3. Cruzamento de dados entre as variáveis relação das aulas teóricas/práticas e segurança para atuar

\begin{tabular}{lcccc}
\hline Relação aulas teóricas/práticas & \multicolumn{4}{c}{ Segurança para atuar } \\
\cline { 2 - 5 } & Seguro/sozinho & Seguro/supervisionado & Inseguro & Total \\
\hline Satisfatória & 45 & 29 & 0 & 74 \\
Não satisfatória & 24 & 28 & 10 & 62 \\
\hline Total & 69 & 57 & 10 & 136 \\
\hline
\end{tabular}

Teste de associação do Qui-quadrado: $\chi^{2}=5,991 ;$ g.l. $=2 ; p=0,050$ 
as aulas teóricas e práticas foi considerada satisfatória, possibilitando segurança para atuar, conforme correlação estatística.

Os achados mostram o fonoaudiólogo recém-formado atuante em mais de uma área, corroborando a literatura ${ }^{(9-11)}$. A clínica foi o segmento mais pretendido, enquanto a perícia se destaca como sendo o menos desejado. Embora os dados da presente pesquisa não permitam uma análise mais detalhada quanto a esse fato, talvez possamos associar a maior pretensão pela clínica, assim como a menor pretensão pela perícia, aos fatos históricos, uma vez que os conhecimentos ligados à formação vêm propondo, ao longo dos anos, o direcionamento para o atendimento terapêutico fonoaudiológico. Apesar de ser constatado em conteúdo programático dos cursos de Fonoaudiologia, desde a ampliação do currículo mínimo(1), o direcionamento para um profissional mais atento às necessidades da sociedade, a atuação em alguns campos parece ser ainda pouco conhecida, uma vez que os sujeitos têm percepção pouco clara sobre alguns segmentos de atuação, como em perícia, por exemplo. Em princípio, o fonoaudiólogo é o profissional habilitado para o exercício da Perícia em Voz, criminal ou trabalhista ${ }^{(13,15)}$. Vale ressaltar que, nos trabalhos anteriores $^{(8-11)}$, esse segmento não aparece mencionado.

A Linguagem foi considerada a área que melhor subsidiou a formação, enquanto que a Saúde Coletiva foi apontada como a que trouxe menos subsídio. Embora não tenha sido questionada a razão de tais apontamentos, o destaque dessas duas áreas talvez possa ser explicado frente à evolução da profissão. A Linguagem é a área que acompanha a história da Fonoaudiologia desde sua idealização na década de 30, motivada pela preocupação de educadores e médicos com crianças em idade escolar, sendo uma área amplamente conhecida e abordada nos cursos oferecidos ${ }^{(16)}$. A Saúde Coletiva refere-se a um campo relativamente novo, reconhecido como especialidade e inserido nos currículos a partir de $2006^{(14)}$. As instituições educacionais têm, a partir dessa data, a oportunidade de formar profissionais comprometidos com a Saúde Coletiva e com a cidadania, favorecendo o crescimento de oportunidades de prestação de serviço à sociedade. Considerando-se que os sujeitos desta pesquisa são formados em 2005/2006, parece justificável que tal área tenha sido apontada como a que pouco subsidiou a formação. É possível que, devido ao período de formação proposto por este estudo, a área de Saúde Coletiva talvez não tenha sido abordada satisfatoriamente pelas matrizes curriculares.

Continuar se atualizando é desejo de grande parte dos sujeitos. A especialização apresentou-se como a continuidade mais desejada, corroborando alguns trabalhos anteriores ${ }^{(8-11)}$ que investigaram fonoaudiólogos durante os cursos de formação continuada, mostrando que, nas datas das pesquisas, havia menor número de fonoaudiólogos com formação strictosensu do que em lato-sensu ${ }^{(8-11)}$. Em particular, o estudo desenvolvido na Paraíba mostrou que não havia qualquer fonoaudiólogo com formação stricto-sensu ${ }^{(11)}$.

A presente pesquisa aponta maior interesse dos participantes pela especialização em detrimento do mestrado e, em especial, do doutorado. Apesar de os resultados aqui demonstrados não permitirem associações diretas, alguns aspectos valem ser comentados. O primeiro aspecto refere-se às dificuldades de acesso do fonoaudiólogo do estado do Rio de Janeiro aos cursos de pós-graduação stricto sensu. Atualmente, no Rio de Janeiro, existe um único mestrado em Fonoaudiologia recomendado pela Coordenação de Aperfeiçoamento de Pessoal de Ensino Superior (CAPES), fato que pode ter contribuído para a menor intenção de procura dos participantes por esse tipo de continuidade em sua formação profissional. Soma-se ao fato que tal mestrado é oferecido por uma instituição particular, o que demanda disponibilidade financeira. Em nível de doutorado, até a presente data, nenhuma instituição pública ou privada oferece tal curso, levando os fonoaudiólogos residentes no estado do Rio de Janeiro a optarem por doutorados em áreas afins (Lingüística, Educação, entre outros), ou por doutorados em outros estados. A opção por áreas afins pode significar um investimento amplo em termos de conhecimento. Por sua vez, a opção por cursar o doutorado em outro estado implica viagens frequentes, o que exige disponibilidade ainda maior, tanto no aspecto financeiro quanto de tempo. Em contrapartida, os cursos de especialização no mesmo estado apresentam uma oferta consideravelmente maior e requerem disponibilidade financeira mais modesta e de tempo mais reduzido. Na medida em que os dados dizem respeito a fonoaudiólogos com pouco tempo de formados, que concluíram a graduação nos anos de 2005 e 2006, outro aspecto a ser comentado associa-se à preferência dessa população do estado do Rio de Janeiro quanto ao segmento de trabalho. Parece razoável que um perfil mais clínico, como o apontado neste estudo, guie o profissional a cursos de especialização que oferecem um aprofundamento especifico com maior subsídio em relação a dados de diagnóstico e técnicas aplicáveis à prática clínica. $\mathrm{O}$ interesse em buscar a atualização dos conhecimentos por meio de cursos de especialização pode sugerir a conscientização do profissional quanto à necessidade da tal formação e aprimoramento, principalmente voltado para a atuação clínica, segmento mais pretendido pela população aqui estudada. Os cursos de mestrado e doutorado são direcionados para profissionais que pretendem seguir carreira acadêmica, segmento menos procurado conforme dados do presente estudo.

Os resultados que apontaram o segmento clínica, com atuação em consultórios como o mais pretendido, podem estar associados a uma possível noção idealizada do fonoaudiólogo recém-formado de que a atuação em consultórios requeira menor esforço inicial por apresentar uma demanda clara, com um trabalho reconhecido pela sociedade. Já a atuação no segmento empresarial, via emprego formal ou prestação de serviços, depende da existência de vagas, da busca pelas empresas e divulgação da existência do trabalho, além de ter a demanda forçada por leis de segurança e medicina do trabalho (Normas Regulamentadoras 7 e 14 aprovadas pela portaria $\mathrm{n}^{\circ} 3.214$, de 8 de junho de 1978), e cuja fiscalização cabe aos órgãos responsáveis pela promoção da segurança e saúde do trabalhador. A literatura aponta a rede hospitalar como o ambiente de trabalho que emprega o menor número de fonoaudiólogos. Os dados do estado de São Paulo mostram indicador de $4 \%^{(8)}$, enquanto que na região Sul os dados mostram apenas $2 \%^{(9)}$, bem como em localidades menores como a cidade de São José dos Campos ${ }^{(10)}$. Da mesma forma, 
a literatura mostra que a indústria apresenta indicadores de emprego bastante reduzidos com $2 \%$ em São Paulo e região $\mathrm{Sul}^{(8-9)}$ e $4 \%$ em São José dos Campos ${ }^{(10)}$. Os achados do presente trabalho apontam o conjunto formado por hospitais, indústrias e empresas como os ambientes de trabalho de acesso mais difícil, segundo a opinião dos fonoaudiólogos recém-formados, corroborando os dados de oferta nesses locais pontuados por esses trabalhos anteriores ${ }^{(8-10)}$. Vale salientar que os dados referidos em tais estudos já podem se encontrar defasados, uma vez que esses ambientes de trabalho tiveram maior abertura e aceitação profissional nos últimos anos.

No presente estudo, a universidade e a escola foram apontadas pelos participantes como o segundo conjunto de locais de trabalho considerados de fácil acesso. Entretanto, os achados relacionados à docência não mostram a mesma convergência. A literatura ${ }^{(9-1)}$ apresenta índices relativamente baixos de fonoaudiólogos docentes: $3,25 \%^{(9)} ; 3,75 \%{ }^{(10)}$; $1 \%{ }^{(11)}$. Talvez essa reduzida porcentagem de fonoaudiólogos atuantes em docência indique que as instituições de ensino não apresentam condições de absorver estes profissionais. Uma vez que ocorrem modificações constantes quanto à oferta $\mathrm{e}$ demanda de trabalho, parecem ser necessários novos estudos voltados para esse tipo de informação quanto ao mercado de trabalho fonoaudiológico e associações pertinentes.

Não obstante o julgamento ou pretensão quanto ao campo de trabalho, os resultados deste estudo mostraram que a maioria dos fonoaudiólogos formados há dois e três anos encontra-se atuando profissionalmente. A porcentagem de $87 \%$ parece consideravelmente alta, podendo não refletir a realidade de forma geral.

Ressalte-se que a generalização destes achados deve ser realizada com a devida cautela, uma vez que, embora estejam descritos na literatura ${ }^{(13)}$, os conceitos de segmentos, áreas e locais de atuação podem gerar confusão quando aplicados ao saber comum, seja por falta de informação do profissional ou desconhecimento por demanda muito reduzida em alguns segmentos e áreas nos diferentes estados do país. Como limitação do presente estudo, vale ainda salientar que o segmento que se refere à gestão não foi contemplado. Sugerese, portanto, que novos estudos sejam conduzidos incluindose tal segmento de atuação, assim como a verificação do conhecimento que os profissionais recém-formados têm quanto às suas possibilidades de atuação, buscando-se identificar a necessidade de divulgação dessas pelos órgãos competentes que regem a profissão.

As constantes atualizações curriculares e modificações no mercado de trabalho da Fonoaudiologia propiciam constantes movimentos no panorama de interesse do profissional recémformado e ofertas de permanente atualização do conhecimento. Dessa forma, a percepção do fonoaudiólogo quanto a sua formação, intenção profissional e busca por atualização pode apresentar mudanças contínuas a serem verificadas, a fim de permitir discussões produtivas que possam dar suporte às futuras modificações e ajustes dos programas de graduação e de pós-graduação, buscando contemplar a evolução da profissão.

\section{CONCLUSÃO}

Os fonoaudiólogos entrevistados consideraram-se atendidos em suas expectativas quanto à sua formação e seguros frente aos conhecimentos adquiridos. A Linguagem foi considerada a área que melhor subsidiou sua formação, enquanto que Saúde Coletiva foi considerada aquela que menos atendeu às expectativas.

Grande parte dos fonoaudiólogos participantes pretende atuar em consultórios e clínicas particulares. A indústria, empresa e hospital foram enfocados como os locais de acesso mais difícil, segundo a opinião dos fonoaudiólogos recémformados.

A atualização dos conhecimentos é pretendida, sendo que os cursos de especialização apresentaram-se como os de maior interesse.

\begin{abstract}
Purpose: To investigate the perceptions of newly graduated Speech and Language Pathologists (SLP) from the state of Rio de Janeiro regarding their academic formation, professional goals and search for knowledge updates. Methods: SLPs graduated from universities located at the state of Rio de Janeiro during the years of 2005 and 2006 participated in the study. A questionnaire with ten closed questions, developed for the present study and validated through a pilot study, was applied. Data were statistically analyzed comparing different variables. Results: It was observed that the majority of the SLPs interviewed considered that their expectations were met and felt safe about the knowledge acquired $(\mathrm{p}<0.001)$. These professionals wish to act mostly in clinics $(89 \%)$, and intend to continue their studies through lato sensu programs (70\%). Language was considered the area in which they received the best support during their training (68\%), while Public Health was the area in which they considered to have received less support (72\%), and this difference was statistically significant. Industry was considered the most difficult workplace to be accessed by SLPs ( $\mathrm{p}<0,05)$. Conclusion: Newly graduated SLPs from the state of Rio de Janeiro considered to have met their expectations regarding their formation, especially in the Language area, and showed more interest for the clinical setting. Lato sensu programs were the most attractive to the population studied for updating their knowledge.
\end{abstract}

Keywords: Professional training; Speech, language and hearing sciences/education; Education, continuing; Job market 


\section{REFERÊNCIAS}

1. Conselho Nacional de Educação. Câmara de Educação Superior Resolução CNE/CES 5, de 19 de fevereiro de 2002. Institui Diretrizes Curriculares Nacionais do Curso de Graduação em Fonoaudiologia [citado 2010 Jan 20]. Disponível em: http://www.fonoaudiologia.org. $\mathrm{br} / \mathrm{htm} / \mathrm{leis} 02 . \mathrm{jsp}$

2. Collares CA, Moysés MA, Geraldi JW. Educação continuada: a política das descontinuidades. Educ Soc [Internet].1999 [citado 2010 Jan 20]; 20(68):202-219. DOI 10.1590/SO101-73301999000300011. Disponível em: http://www.scielo.br/pdf/es/v20n68/a11v2068.pdf

3. Moll KL. Training programs in logopedics. Folia Phoniatr Logop. 1983;35(5):198-219.

4. Silva AP, Massi GA. Práticas fonoaudiológicas: uma análise histórica. Pró-Fono.1999;11(1):150-2.

5. Ferreira LP. Speech therapy in Brazil: forty years of existence, two decades of recognition. Folia Phoniatr Logop. 2002;54(2): 103-5.

6. de Meis L, Velloso A, Lannes D, Carmo MS, de Meis C. The growing competition in Brazilian science: rites of passage, stress and burnout. Braz J Med Biol Res. 2003;36(9):1135-41.

7. Behlau M, Gasparini G. Education of speech-language pathologists and audiologist in Brazil. Folia Phoniatr Logop. 2006;58(1):14-22.

8. Conselho Regional de Fonoaudiologia do Estado de São Paulo. $2^{\text {a }}$ região Perfil do fonoaudiólogo no estado de São Paulo. São Paulo: Conselho Regional de Fonoaudiologia do Estado de São Paulo. 2a região; 1997.
9. Ribas A, Teixeira SB, Luna RCM, Ristow SH, Berberian AP, Massi GA. Perfil do fonoaudiólogo na região sul do Brasil. J Bras Fonoaudiol. 2001;2(6):29-35.

10. Stefaneli FR, Monteiro KD, Spinelli RL. Perfil do fonoaudiólogo na cidade de São José dos Campos. Rev CEFAC. 2004;6(1):101-05.

11. Almeida LR, Guedes AC, Pereira HS, Neves VD, Nunesmaia MM, Nunesmaia HG. Característica da formação do fonoaudiólogo no estado da Paraíba. Rev Fono Brasil. 2005;3(1):1-3.

12. Perfil do fonoaudiólogo. Dialogando, informe semanal do CRFa1 [internet]. Conselho Regional de Fonoaudiologia $1^{\mathrm{a}}$ região[citado em 2007Abr4, 2007Mai11 e2007Ag1].

13. Conselhos Federal e Regionais de Fonoaudiologia. Classificação brasileira de procedimentos em fonoaudiologia. $3^{\mathrm{a}}$ ed. [Internet] [citado 2010 Jan 20. Disponível em: http://www.fonoaudiologia.org.br/ discovirtual/pubdownload/cbpfa2ed.pdf

14. Conselho Federal de Fonoaudiologia. Resolução n. 320, de 17 de fevereiro de 2006. Dispõe sobre as especialidades reconhecidas pelo Conselho Federal de Fonoaudiologia, e dá outras providências [internet] [citado 2010 Jan 20. Disponível em: http://www.fonoaudiologia.org.br/ servlet $/$ ConsultaLegislacao acao $=\mathrm{V} \&$ leiId $=35$

15. Sávio DF. A fonoaudiologia no campo empresarial. Fono Atual. 2004;7(29):79-81.

16. Canongia MB, Caraciki AM, Cardoso I. A história da fonoaudiologia no estado do Rio de Janeiro. Rio de Janeiro: Lovise; 2004. 